\title{
Les écoles communautaires dans les zones rurales de Tanzanie
}

The development of community schools in rural zones of Tanzania

El desarrollo de las escuelas comunitarias en las zonas rurales de Tanzania

\section{Nathalie Bonini}

\section{OpenEdition}

\section{Journals}

Édition électronique

URL : http://journals.openedition.org/ries/2279

DOI : $10.4000 /$ ries. 2279

ISSN : 2261-4265

Éditeur

Centre international d'études pédagogiques

Édition imprimée

Date de publication : 1 avril 2012

Pagination : 83-92

ISBN : 978-2-85420-594-7

ISSN : 1254-4590

\section{Référence électronique}

Nathalie Bonini, «Les écoles communautaires dans les zones rurales de Tanzanie », Revue

internationale d'éducation de Sèvres [En ligne], 59 | avril 2012, mis en ligne le 06 février 2015, consulté le 01 mai 2019. URL : http://journals.openedition.org/ries/2279 ; DOI : 10.4000/ries.2279 


\section{Les écoles communautaires dans les zones rurales de Tanzanie}

\section{Nathalie Bonini}

Depuis sa création en avril 1964, la République unie de Tanzanie (née de l'union entre le Tanganyika et Zanzibar) ${ }^{1}$ a favorisé le développement de l'école rurale. Dirigé jusqu'en 1985 par un ancien instituteur, Julius K. Nyerere, le pays a parfois été présenté comme un modèle africain en matière d'éducation. Selon la philosophie de "l'éducation pour l'autosuffisance " (education for self reliance - ESR) qui a orienté la politique éducative tanzanienne depuis la déclaration d'Arusha en 1967 jusqu'au milieu des années 1980, la primauté fut donnée à l'essor de l'éducation de base, considérée comme le moteur du développement et l'un des piliers de la construction d'un État socialiste.

\section{ÉdUCATION RURALE ET SOCIALISME}

Le développement de l'école rurale répondait à un double objectif : celui d'éduquer le plus grand nombre - une très large majorité de la population résidant en zone rurale - et celui de former des futurs paysans, l'agriculture constituant alors la base du développement économique du pays. À côté des enseignements fondamentaux et de l'éducation politique propageant des valeurs socialistes et d'unité nationale, l'agriculture était enseignée et pratiquée dans l'enseignement primaire et secondaire. Toutes les écoles rurales étaient donc, dans l'idéal, pourvues d'un champ cultivé par les élèves aidés de leurs enseignants, ce qui leur fournissait une expérience pratique des travaux agricoles en même temps qu'une source alimentaire répondant ainsi à l'objectif de l'autosuffisance. De même, pour relier l'éducation scolaire aux activités économiques et sociales, et "mettre l'école à portée de tous les élèves », les écoles rurales étaient préférentiellement implantées dans les villages communautaires connus sous le nom de villages ujamaa. D'abord mis en place sur la base du volontariat, le regroupement des citoyens dans ces collectivités autonomes ujamaa est devenu plus systématique au début des années 1970, puis obligatoire de 1973 à 1976, avant que cette politique de "villagisation » ne soit abandonnée cette même

1. Seule la situation de la Tanzanie continentale sera abordée dans cet article. 
année ${ }^{2}$. Symbole et support de l'unité nationale, le swahili - déjà utilisé dans certaines écoles coloniales ou missionnaires - devient la langue d'instruction unique à l'école primaire, tandis que l'anglais constitue la langue d'enseignement dans les niveaux secondaire et supérieur ${ }^{3}$.

\section{LA NOUVELLE GOUVERNANCE}

La fin des années 1980 est marquée par un changement d'orientations éducatives dicté dans une large mesure par les bailleurs de fonds à l'origine de l'implantation de plans d'ajustement structurel. Si la politique de l'ESR est alors abandonnée, la suprématie accordée au niveau primaire n'est pas entamée ; au-delà de ce niveau, le système demeure très sélectif jusqu'à la fin des années quatre-vingt-dix.

La décentralisation de la gestion et de l'administration des écoles aux niveaux des autorités locales et des communautés (du canton) représente l'une des mesures phares de ce plan. Désormais, les rôles se repartissent ainsi : le ministère de l'éducation gère les programmes scolaires, répartit les enseignants sur le territoire, contrôle le budget et finance la viabilisation des bâtiments scolaires nouvellement construits, tandis que l'administration de district se charge de déployer le contingent d'enseignants attribué au district dans les différentes écoles et de coordonner l'implantation de nouvelles écoles. L'administration de canton, quant à elle, est chargée d'identifier les zones de construction de nouvelles écoles, de rassembler les ressources physiques et financières de la communauté pour les construire et de mobiliser les parents d'élèves pour favoriser les inscriptions et l'assiduité des élèves.

C'est selon ce schéma d'organisation et de financement que sont créées la plupart des écoles primaires et secondaires que l'on nomme alors «communautaires » depuis la fin des années quatre-vingt-dix. Ces écoles communautaires sont publiques mais initiées, construites et en grande partie financées par les habitants eux-mêmes. Au niveau de l'enseignement primaire, ces écoles communautaires viennent enrichir le nombre d'écoles gouvernementales et ne diffèrent de ces dernières que par leur mode de financement initial (par la communauté), tandis qu'au niveau secondaire, la différence est également une distinction de statut. Le parc des écoles secondaires gouvernementales est en effet constitué de deux types d'établissements : les écoles à rayonnement national ou régional, qui sont souvent plus anciennes et généralement situées en villes

2. En 1976, on comptait environ huit mille villages ujamaa regroupant $85 \%$ de la population.

3. Le système éducatif tanzanien se compose de deux années d'enseignement pré-primaire, sept années de primaire, quatre années de cycle inférieur d'enseignement secondaire, deux années de cycle supérieur d'enseignement secondaire et trois années ou plus d'enseignement supérieur. Pour une analyse plus détaillée, voir Bonini (2003). 
ou en périphérie, et les écoles communautaires, moins prestigieuses, qui n'offrent pour la plupart que les quatre premières années d'enseignement du cycle secondaire ${ }^{4}$ et dont le recrutement est essentiellement local.

\section{DISPARITÉ DE L'OFFRE ÉDUCATIVE DANS LES ZONES RURALES}

Malgré une urbanisation croissante ${ }^{5}$, la population rurale représente environ $70 \%$ de la population du pays et le secteur de l'agriculture occupe plus de $70 \%$ des actifs. Les zones rurales concentrent par ailleurs l'immense majorité des personnes pauvres de Tanzanie (Wedgwood, 2005), dont le produit intérieur brut (PIB) est lui-même l'un des plus bas du monde. Toutefois, il existe une grande disparité tant sur le plan socio-économique qu'en termes de peuplement ou encore de taux de scolarisation entre les différentes zones rurales du pays. Les régions rurales du nord-ouest (autour du Lac Victoria) et du nord (autour du Kilimanjaro), en majorité peuplées d'agro-pasteurs ou d'agriculteurs engagés notamment dans la culture du maïs, de bananes, du café ou du thé, enregistrent les plus fortes densités de population. Si elles n'atteignent pas celles des montagnes du Rwanda voisin, les régions rurales montagneuses tanzaniennes sont en moyenne plus densément peuplées que les grandes plaines semi-arides ou arides du Nord et du Centre, qui abritent surtout des populations agropastorales ou pastorales.

Ces différences de peuplement résultent en partie des conditions climatiques, qui ont elles-mêmes influencé les politiques de développement territorial pendant la colonisation et après l'indépendance. Tant en milieu urbain que dans les zones rurales, les écoles ont majoritairement été implantées dans les endroits fortement peuplés. Il en est ainsi par exemple de la région de Dar es Salaam, à dominante urbaine, et de celle du Kilimanjaro, à dominante rurale, qui enregistrent des niveaux de scolarité supérieurs aux autres régions. Ainsi en 2004, la proportion d'hommes et de femmes n'ayant jamais été scolarisés était respectivement de $12 \%$ et de $14 \%$ dans la région de Dar es Salaam et de $12 \%$ et $15 \%$ dans celle du Kilimanjaro, contre $44 \%$ et $55 \%$ dans la région de Tabora au Centre-Ouest du pays (NBS, 2005, p. 13).

$\mathrm{Du}$ fait de leur isolement et de leur accès difficile, les zones rurales les plus reculées ont pendant longtemps disposé d'une faible couverture scolaire. L'amélioration de l'infrastructure scolaire dans ces endroits est considérée comme l'un des moyens d'atteindre la scolarisation universelle, et constitue de ce fait un objectif majeur des plans de développement de l'éducation élaborés depuis la fin

4. Sur le modèle du $O$-level et $A$-level britannique, l'enseignement secondaire est divisé en deux cycles : le cycle inférieur « lower secondary » d'une durée de quatre ans (de form 1 à form 4) puis le cycle supérieur upper secondary de deux ans (forms 5 et 6).

5. La proportion de personnes résidant dans les zones urbaines était de $6 \%$ en 1967, de $18 \%$ en 1988 et de $23 \%$ en 2002 (NBS, 2005, p. 2). 
des années quatre-vingt-dix. Dans ce dispositif éducatif décentralisé, la participation des parents, qui représente une source de financement supplémentaire pour la construction des écoles, est également perçue comme favorisant leur implication dans la scolarité de leurs enfants et, ainsi, leur taux de scolarisation.

Si l'on observe des différences d'équipement et de scolarisation entre les villes et les zones rurales ${ }^{6}$, à l'intérieur même des zones rurales, le rythme des constructions d'écoles et leur qualité varie d'un endroit à l'autre. Pour être enregistrée comme école publique et être pourvue en enseignants et en matériel pédagogique par le gouvernement, l'école communautaire doit en principe comprendre au minimum une salle de classe par niveau, des sanitaires, des locaux pour l'administration et des habitations pour les enseignants - le tout devant être meublé -, auxquels s'ajoutent un laboratoire pour les écoles secondaires et au minimum deux dortoirs (pour les garçons et pour les filles) dans le cas des internats. L'école doit disposer d'eau, de l'électricité et se situer à proximité d'un dispensaire et d'une route.

Or nombre de cantons, notamment ceux situés dans les zones rurales isolées qui abritent souvent les familles les plus pauvres, remplissent difficilement les conditions nécessaires à l'ouverture des établissements ou ne les remplissent que provisoirement, le temps de leur inscription sur les registres officiels (Galabawa 2001, p. 38). Faute de fonds, toutes les écoles programmées ne peuvent être construites, dans la mesure où les financements de l'État n'interviennent qu'une fois le gros œuvre réalisé. Ainsi, les zones rurales les plus reculées, qui sont précisément celles ciblées par le plan de développement des écoles communautaires, sont aussi celles qui ont le plus de difficultés à en financer la construction - et surtout à les entretenir ensuite - et, comme on le verra ci-dessous, à retenir les enseignants et les élèves.

En revanche, les zones rurales les plus favorisées ont accru leur offre éducative par la création d'écoles communautaires. À titre d'exemple concernant le niveau secondaire, la région du Kilimanjaro, où la supériorité en matière d'éducation scolaire remonte aux toutes premières heures de la colonisation, qui comptait en 2006 environ 1,5 million d'habitants, comptait deux cent vingt-deux écoles secondaires dont neuf gouvernementales et cent vingt-cinq communautaires (dont trente-cinq proposaient des enseignements de la première à la sixième année du secondaire), tandis qu'à la même époque, la région de Rukwa qui comprenait un nombre d'habitants légèrement inférieur (1,3 million) disposait de cinquante-trois écoles secondaires (deux gouvernementales et quarantedeux communautaires) qui n'allaient pas au-delà de la quatrième année, et celle de Kagera était pourvue de cent treize écoles secondaires (cinq gouvernementales et quatre-vingt-cinq communautaires) dont six fournissant les six années d'enseignement pour environ 2,3 millions d'habitants. Cette suprématie numérique de rurales tandis qu'il était respectivement de $18,9 \%$ et de 3,1 \% pour le niveau secondaire (NBS, 2005, p. 16). 
certaines régions en matière d'écoles secondaires s'accompagne d'une meilleure réputation et d'une plus grande qualification (c'est-à-dire d'un plus grand nombre de personnels qualifiés) des établissements qui sont par ailleurs plus nombreux à proposer un enseignement incluant les six années du secondaire ${ }^{7}$.

Cette disparité en matière d'offre éducative secondaire entre les différentes régions du pays a, ces toutes dernières années, conduit le gouvernement à centrer ses efforts financiers en direction des zones les moins dotées. Ainsi, en 2009, pratiquement tous les cantons du pays étaient pourvus d'une école secondaire et les régions qui avaient moins de cinquante écoles secondaires quatre ans plus tôt ont vu leur nombre d'écoles doubler, à l'image de celles de Lindi (passée de quarante-deux écoles à cent dix-sept) de Tabora (de quarante-six à cent soixante-quatre) ou de Rukwa (de quarante-six à cent trois) (URT, 2010, p. 11).

\section{LA MOINDRE QUALITÉ DES ÉCOLES DANS LES ZONES RURALES RECULÉES}

Le développement rapide de l'infrastructure scolaire sur l'ensemble du territoire via les constructions d'écoles communautaires s'est souvent fait au détriment de la qualité d'enseignement. Nombreux sont les rapports et études ${ }^{8}$ qui soulignent la moindre qualité, en termes de locaux, de matériel pédagogique, de ratio enseignant/élèves, de niveau de formation des enseignants, dans les espaces ruraux et notamment dans les zones les plus isolées.

À ces mauvaises infrastructures scolaires s'ajoute le fait que les ménages qui composent ces cantons n'ont souvent pas les moyens de payer les frais de scolarité de leurs enfants. Que l'école primaire soit gratuite depuis la fin de l'année 2001 n'empêche pas les frais annexes, tels l'achat d'uniforme, les contributions «volontaires » que les écoles demandent parfois aux parents, le matériel pédagogique - théoriquement fourni par l'État mais pour lesquels les parents sont régulièrement sollicités - et d'une façon plus générale, le coût d'opportunité que représente la scolarisation d'un enfant qui, lorsqu'il est à l'école, n'aide plus dans les champs ou à garder le bétail. Ces frais sont amplifiés au secondaire par les frais de scolarité - qui s'élevaient jusqu'en 2004 à 70000 TSh (shilling tanzanien) pour les internats et 40000 TSh pour les externats (Wedgwood, 2005, p. 13), mais qui ont été réduits à 20000 TSh pour les externats (environ 15 USD) en janvier 2005 (URT, 2010, p. 9) -, par les frais d'examens, les coûts de transport, lorsque l'établissement fréquenté est éloigné, ou l'argent de poche, notamment pour les enfants internes qui doivent payer eux-mêmes de nombreux frais annexes.

7. Les écoles offrant les six années de second cycle ne représentaient à la fin des années quatre-vingt-dix, que $10 \%$ des écoles secondaires (Lassibille et al., 2000, p. 8).

8. Cf. Galabawa, 2001 ; MoEVT , 2010 ; NBS, 2005 ; Sumra S. \& Rajani R., 2006 ; Wedgwood, 2005 et Woods, 2008. 
Le manque de ressources, mais également l'économie rurale agricole ou pastorale qui nécessite la participation des enfants de façon régulière ou saisonnière influe sur l'assiduité des élèves et sur leur maîtrise des apprentissages. Par ailleurs, en l'absence de matériel ad hoc, cette dernière repose souvent sur la récitation ou la mémorisation de ce que dicte l'enseignant. Au niveau primaire, la pénurie d'enseignants et/ou de locaux, comme la volonté d'augmenter le taux de scolarisation, ont accru la proportion d'écoles pratiquant le double flux depuis les années 2000, certains élèves étant scolarisés le matin et d'autres l'après-midi.

Cette moindre qualité de l'enseignement a pour conséquence un taux de réussite inférieur à l'examen de fin de cycle primaire (PLSE) des élèves ruraux, ou l'obtention de moins bonnes notes lorsqu'ils y parviennent ${ }^{9}$. Selon Wedgwood, en 1998, le taux de réussite à l'examen PLSE était de $17 \%$ dans les zones rurales contre $29 \%$ en zone urbaine (Wedgwood, 2005, p. 13). Ces moindres performances influent sur le type d'établissements secondaires fréquentés, dans la mesure où les places dans les écoles secondaires gouvernementales les plus prestigieuses sont essentiellement attribuées en fonction des notes obtenues à l'examen. Ainsi, les élèves scolarisés dans une école primaire rurale qui parviennent à intégrer le secteur secondaire public le font, la plupart du temps, au sein d'écoles communautaires moins réputées et moins bien dotées que les écoles publiques régionales. En effet, les difficultés qui prévalent dans l'enseignement primaire sont accentuées dans les écoles secondaires communautaires, qui nécessitent des moyens supplémentaires et connaissent une pénurie d'enseignants plus importante.

\section{LE MANQUE D'ENSEIGNANTS EN MILIEU RURAL}

Du fait de l'augmentation exponentielle des établissements scolaires et notamment des écoles secondaires et de la faiblesse de formation des enseignants, le taux de scolarisation à ce niveau étant demeuré très faible pendant de nombreuses années, la Tanzanie fait face à une pénurie d'enseignants particulièrement criante en milieu rural. Les zones rurales rebutent en effet de nombreux enseignants et notamment les plus qualifiés ${ }^{10}$, qui préfèrent s'installer en ville ou en périphérie pour un salaire équivalent. Lorsqu'ils sont en poste dans ces écoles reculées, les enseignants doivent parfois s'occuper de plusieurs classes,

9. Seuls les élèves ayant réussi cet examen de fin de cycle primaire peuvent être inscrits dans les écoles secondaires gouvernementales.

10. La formation des enseignants se fait soit au niveau du Teacher Training Collège - TTC), soit à l'université. Les enseignants des écoles primaires et du premier cycle du secondaire ont généralement intégré le TTC après sept années de cycle primaire (ils ont alors le grade $C$ après deux années de formation au TTC ou le grade $B$ après quatre années de formation au TTC) ou après avoir terminé les quatre années de premier cycle du secondaire (auxquels s'ajoutent deux ans de formation au TTC, ce qui leur donne le grade A). Les enseignants de secondaire et a fortiori ceux qui enseignent dans le cycle supérieur du secondaire ont généralement intégré l'université après avoir réussi l'examen à la fin de la sixième année du secondaire. 
soit en attendant la nomination d'un collègue, soit en raison de son absence pour motifs personnels (maladie, contraintes familiales), administratifs (voyage dans le chef-lieu de canton pouvant durer plusieurs jours, pour les besoins de l'école ou pour percevoir le salaire) ou pour d'autres raisons liées à leur situation financière précaire et à leur implication dans des activités complémentaires. En dehors du manque d'aménagements et de l'accès difficile par manque d'infrastructures routières ou de transports dans ces zones isolées, l'environnement scolaire lui-même décourage certains enseignements dans la mesure où, construites rapidement et avec peu de moyens, nombreuses sont les écoles qui ouvrent avant d'être terminées, ne disposent pas d'habitation pour les enseignants en nombre suffisant, manquent de matériel pédagogique et, dans les zones les plus reculées, d'accès à l'eau ou à l'électricité.

Ainsi, la pénurie générale d'enseignants est accentuée par le fait que ceux qui sont disponibles tendent à se détourner des écoles rurales (URT, 2010, p. 33). En 2007, le ratio moyen élèves/enseignant était de soixante-trois élèves pour un enseignant, avec une forte disparité entre les zones urbaines pour lesquelles il était de quarante-six élèves pour un enseignant et les zones rurales qui, elles, comptaient un enseignant pour soixante-et-onze élèves (MoEVT, 2010). Signalons que, contrairement à ce que l'on peut parfois rencontrer dans les zones urbaines ou dans certaines zones rurales densément peuplées, ce mauvais ratio n'est pas, dans ce cas, dû à la surpopulation scolaire mais bien à l'absence d'enseignants.

Selon différentes études, dans nombre d'écoles secondaires communautaires créées au milieu des années deux mille, le personnel enseignant est constitué de personnes ayant quitté l'école après la quatrième année du secondaire sans avoir obtenu de diplôme (Wedgwood, 2005 ; Woods, 2008). Leur maîtrise des disciplines scientifiques et de l'anglais, qu'ils doivent utiliser pour enseigner, est généralement moindre que celle des enseignants formés à l'université, que l'on rencontre plus souvent dans les écoles urbaines. Comme le note Wedgwood, contrairement à ce qui avait été affiché dans le plan de 2004, peu de mesures incitatives ont été déployées pour encourager les enseignants à occuper des postes dans les zones reculées du pays. Les postes sont aussi pourvus par des enseignants stagiaires en première année de formation. Il en résulte un fort turnover des enseignants, ce qui nuit à la qualité de l'enseignement dispensé (Wedgwood, 2005).

Comme le soulignent plusieurs auteurs, dont Wedgwood (2005) qui a réalisé une enquête de terrain dans les régions rurales du district de Morogoro, et comme nous avons pu le constater également dans les régions reculées du Nord du pays, la vacance des postes d'enseignants dans ces établissements ruraux, comme la médiocrité des infrastructures scolaires elles-mêmes, influent sur l'absentéisme et sur le taux d'abandon des élèves et peuvent provoquer la rancœur de leurs parents qui ont participé financièrement à la construction de l'école. 


\section{LE CAS DES ÉCOLES RURALES DES ZONES PEUPLÉES PAR LES MASSAÏ}

La situation des zones reculées peuplées par les Massaï que nous avons étudiées lors de nos enquêtes de terrain au début des années quatre-vingt-dix, puis en 2009, est une illustration des mauvaises conditions de scolarisation fréquentes dans les zones rurales isolées. Les Massaï sont des pasteurs seminomades qui, du fait des sécheresses répétées ces dernières années et de l'aliénation continue de leurs zones de pâturage au profit des terres cultivées et des parcs nationaux, entraînant une situation de surpâturage et, à terme, une diminution de leurs troupeaux, sont de plus en plus contraints d'abandonner leur mode de production pastoral, voire le mode de production agro-pastoral que nombre d'entre eux avaient adopté.

Dans ces conditions de dégradation du pastoralisme, la scolarisation des enfants, qui fut pendant longtemps peu prisée, d'autant qu'elle entrait en concurrence avec les activités quotidiennes de jeunes gardiens de troupeaux, est désormais perçue comme un moyen de diversifier leurs ressources. S'ils demeurent parmi les plus faiblement scolarisés du pays, ils sont aujourd'hui plus nombreux sur les bancs de l'école, y compris secondaire. L'augmentation de l'offre scolaire, passée de soixante-quatorze écoles primaires en 1993, dans les deux districts concernés, à cent quarante-et-un en 2008 et respectivement de deux écoles secondaires (dont une privée) à trente établissements secondaires (dont vingt-quatre publics), la gratuité de l'école primaire depuis 2001, les fortes pressions extérieures en faveur de la scolarisation et la constante dégradation du pastoralisme, entraînant une paupérisation importante et libérant les enfants de la garde du bétail, se conjuguent pour inciter les Massaï à scolariser davantage leurs enfants.

À cela s'ajoute la nécessité de maîtriser le swahili, langue nationale de Tanzanie et moyen de communication avec les non Massaï (pour le commerce, dans les transports, les hôpitaux, les écoles, etc.). C'est d'ailleurs à l'aune de la capacité à enseigner le swahili que l'école primaire est jugée par de nombreux parents d'élèves interrogés ${ }^{11}$. Contrairement à la majorité des enfants tanzaniens, qui parlent une langue maternelle bantoue de la même famille linguistique que le swahili, les Massaï, de langue maternelle nilotique, doivent ainsi apprendre une langue d'une structure totalement différente et, à partir de la troisième année, l'anglais comme langue étrangère, langue qu'ils devront ensuite maîtriser s'ils veulent poursuivre leur scolarité au-delà du primaire.

11. Cette situation était particulièrement vraie dans les années 1990 où les enfants maasai n'étaient pratiquement jamais scolarisés au-delà du primaire, ce qui n'est plus le cas aujourd'hui, même si leur taux de scolarisation au niveau secondaire demeure parmi les plus bas du pays (voir Bonini, 2011). 
Pour autant, leurs conditions de scolarisation demeurent médiocres et les taux d'abandon ou d'échec sont importants. Pour diverses raisons déjà évoquées, il n'est pas rare que les élèves des écoles primaires soient seuls dans leur classe ou sous la direction d'un élève plus âgé qui leur fait réciter leurs leçons oralement et en chœur. Leur travail terminé, les élèves des petites classes qui sont en double flux rentrent chez eux pour laisser la place à ceux qui ont cours l'après-midi, tandis que les autres demeurent dans l'enceinte de l'école lorsqu'ils habitent trop loin pour rentrer. Depuis quelques années, un repas du midi provenant du World Food Programme est distribué dans certaines écoles rurales, ce qui améliore sensiblement la situation des élèves, voire influence leur assiduité à l'école.

$\mathrm{Au}$ niveau des écoles secondaires, nos visites dans trois écoles communautaires de différents cantons ouvertes depuis 2007 et les entretiens réalisés avec les enseignants présents ont révélé des conditions de scolarisation difficiles. Deux ans après l'ouverture des établissements, les bâtiments n'étaient pas terminés et, comme le déplorait l'un des enseignants de l'école secondaire d'Engaruka, qui ne comprenait en 2009 que les trois premières années du cycle inférieur : "Avec l'arrivée de nouveaux élèves l'an prochain, nous serons certainement obligés de transformer l'une des salles de classe en dortoir pour filles la nuit et de ranger les lits pour installer les tables le jour ». En outre, deux des écoles secondaires visitées en 2009 subissaient une pénurie d'eau depuis plusieurs semaines. Dans l'une d'elles, élèves et enseignants avaient passé de nombreuses journées à aller chercher de l'eau avant de décider de creuser une tranchée pour y faire passer un tuyau, activité qui les avait occupés pendant plus de quinze jours, au détriment de leurs cours.

Toutefois, les mauvaises conditions de scolarisation ne sont pas la seule cause de la faiblesse de la scolarisation des enfants massaï : l'incapacité des parents à financer leurs études, notamment au niveau secondaire, ou à se passer d'eux pour les travaux agricoles ou domestiques représente également un facteur décisif. En effet, les parents qui perçoivent la scolarisation comme une alternative au mode de vie pastoral sont souvent ceux qui n'ont pas les moyens de payer les frais qu'engendre la scolarisation secondaire, ce qui limite les perspectives d'emploi.

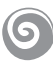

Les récents plans de développement de l'éducation mis en œuvre depuis les années deux mille en Tanzanie ont permis un accroissement sans précédent de l'infrastructure scolaire (tant primaire que secondaire) particulièrement notable dans les zones rurales où celle-ci était relativement faible. Cette meilleure couverture scolaire a favorisé la scolarisation notamment secondaire des enfants et diminué les coûts de transport et d'hébergement qu'engendrait la 
scolarisation post-primaire en dehors du canton. En outre, le fait que cette expansion soit essentiellement due à la création d'écoles communautaires publiques a limité les frais de scolarité - supérieurs dans le secteur privé.

Toutefois, les conditions de scolarisation et la qualité de l'enseignement dispensé dans ces écoles rurales, et notamment dans celles des zones les plus reculées, qui sont souvent également les plus pauvres, demeurent en deçà de celles qui prévalent en ville ou dans les zones plus favorisées. Nombre de ces établissements communautaires ruraux n'ont pas les moyens humains ni matériels de dispenser une éducation de qualité qui puisse favoriser la réussite des élèves aux examens de fin de cycle, et même, selon certains auteurs, l'acquisition de connaissances utiles pour leur vie future (HakiElimu, 2009 ; Sumra \& Rajani, 2006 ; Woods 2008).

\section{BIBLIOGRAPHIE}

BONINI N. (2003) : «Un siècle d'éducation scolaire en Tanzanie », Cahiers d'études africaines, $\mathrm{n}^{\circ} 169-170$, p. 41-62.

BONINI N. (2011) : "Le développement de l'enseignement secondaire en Tanzanie et la scolarisation des Massaï ", Autrepart, 2011/3 n 59, p. 57-74.

GALABAWA C.J. (2001) : "Problématique et évolution de l'enseignement primaire universel (EPU) en Tanzanie ", Biennale ADEA (Arusha, Tanzanie, 7-11 octobre 2001).

HAKIELIMU (2009) : «Annual Report 2009 », http://www.hakielimu.org

LASSIBILlE G., TAN J.-P., SUMRA S. (2000) : "Expansion of Private Secondary Education: Lessons from Recent Experience in Tanzania » Comparative Education Review, Vol. 44, n 1, p. 1-28.

MINISTRY OF EDUCATION AND VOCATIONAL TRAINING (MoEVT) (2010) : Basic Education Statistics in Tanzania, http://educationstatistics.moe.go.tz/moe/.

NATIONAL BUREAU OF STATISTICS (NBS) [Tanzania] \& ORC MACRO, Tanzania Demographic and Health Survey (DHS) 2004-05, October 2005, Dar es Salaam, Tanzania, $381 \mathrm{p}$.

SUMRA S. \& RAJANI R., 2006, Secondary Education in Tanzania: Key Policy Challenges, Working paper $n^{\circ}$ 9, Dar Es Salaam, HakiElimu.

UNITED REPUBLIC OF TANZANIA (URT) (2010) : Secondary Education Development Programme II (July 2010-June 2015), http://moe.go.tz/PDF/SEDP2010.pdf/.

WEDGWOOD R. (2005) : "Post-Basic Education and Poverty in Tanzania » Working Paper Series $\mathrm{n}^{\circ} 1$, Centre of African Studies, University of Edinburgh.

WOODS E. (2008) : "Progress towards EFA in Tanzania », Prospect, Vol. 38, Education for All by 2015: Progress and Challenges, Spinger, p. 425-430. 